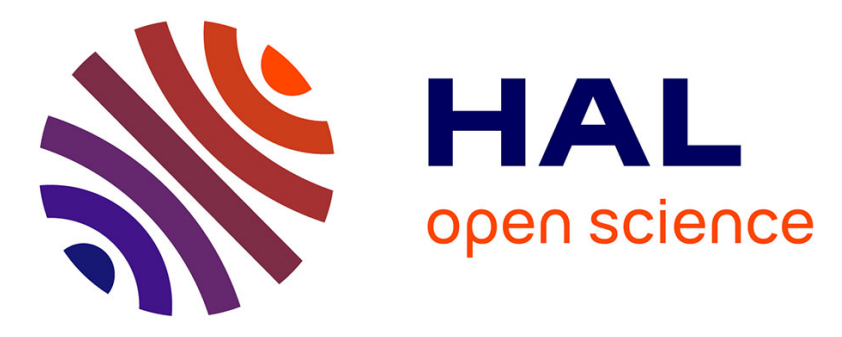

\title{
Micro-fabricated thermal flow-rate sensors: the substrate material impact on the device performance and power consumption
}

Ferdous Shaun, E. Nefzaoui, Frédéric Marty, William César, Tarik Bourouina

\section{- To cite this version:}

Ferdous Shaun, E. Nefzaoui, Frédéric Marty, William César, Tarik Bourouina. Micro-fabricated thermal flow-rate sensors: the substrate material impact on the device performance and power consumption. Microsystem Technologies, 2018, 10.1007/s00542-018-4098-5 . hal-01865930

\section{HAL Id: hal-01865930 \\ https://hal.science/hal-01865930}

Submitted on 2 Sep 2018

HAL is a multi-disciplinary open access archive for the deposit and dissemination of scientific research documents, whether they are published or not. The documents may come from teaching and research institutions in France or abroad, or from public or private research centers.
L'archive ouverte pluridisciplinaire HAL, est destinée au dépôt et à la diffusion de documents scientifiques de niveau recherche, publiés ou non, émanant des établissements d'enseignement et de recherche français ou étrangers, des laboratoires publics ou privés. 


\title{
Micro-fabricated thermal flow-rate sensors: the substrate material impact on the device performance and power consumption
}

\author{
Ferdous Shaun $^{1 *}$, Elyes Nefzaoui ${ }^{1, *}$, Frederic Marty ${ }^{1}$, William Cesar ${ }^{2}$, Tarik Bourouina ${ }^{1}$ \\ ${ }^{1}$ Université Paris-Est, ESYCOM-Lab, ESIEE-Paris, Noisy-Le-Grand, France \\ ${ }^{2}$ Fluigent Smart Microfluidics, 1, Mail du Professeur Georges Mathé, Villejuif, France
}

*ferdousjahan.shaun@esiee.fr, *elyes.nefzaoui@esiee.fr

\begin{abstract}
We report on micro-machined flow-rate sensors as part of autonomous multi-parameter sensing devices for water network monitoring. Three different prototypes of the flow-rate sensors have been designed, fabricated and experimentally characterized. Those sensors are made of identical micrometric platinum resistors deposited on different substrates, made of glass, silicon and micro-structured silicon, with and without insulation layers. The sensors are tested under the anemometric operating scheme. They are experimentally characterized under a water velocity range from 0 to $0.91 \mathrm{~m} / \mathrm{s}$. We show that the glass substrate device is more sensitive and less power-consuming under identical operating condition. We also show that, when silicon is needed as the substrate material, further optimization and design strategies are required. Experimental results are analyzed with respect to Computational Fluid Dynamics simulations with the Finite Element Method.
\end{abstract}

Keywords—-Sensor; MEMS; Flow-rate; Thermal sensor; Micromachined sensor; Water Network Monitoring

\section{INTRODUCTION}

Flow-rate measurement is widely used in various fields including biological and bio-medical applications [1]-[6], industrial processes [7], environmental [8], [9] monitoring and weather forecasting [10]. For instance, flow-rate measurement is particularly important for water distribution systems monitoring not only for measuring water consumption but also for leakage detection. Generally, a water distribution system at an urban scale is a vast network. Consequently, a finely meshed monitoring system based on a sensor network can be quite expensive. Micro-fabrication technologies offer solutions for such a problem with a good meshing/cost tradeoff through cheap lab on chip devices, which can be deployed in large number. During the last decades, the advancement of micro-fabrication techniques enabled the design of low-cost miniaturized sensors optimized for different applications. Extensive reviews can be found in [11], [12]. Such devices offer several advantages: compactness, low cost and low power consumption. In addition, mass production can be considered thanks to the wide range of flow-rates that can be covered by these sensors, from $\mu 1 / \mathrm{min}$ to liter/min [13], [14]. Flow-rate sensors are mainly based on mechanical and thermal transduction phenomena. The device described in the present work belongs to the second category. Thermal flow-rate sensors can be sorted into three categories: calorimetric, anemometric (hot-wire) and time-of-flight (TOF) and () [11], [14]. A Calorimetric sensor detects the fluid flow by measuring temperature distribution around the heater by at least one temperature sensor. A hotwire flow-rate sensors is made of a resistive wire, which transfers heat to the surrounding fluid. The hot wire also acts as a temperature probe. Flow-rate is deduced from the convective cooling rate of the hot wire due to the fluid flow [6],[8].The TOF method is based on measuring the travel time of a thermal pulse from a heater to a temperature sensor. Accordingly, it is obvious that the largest the convective heat flow compared to other heat flows, the largest the sensor sensitivity. Indeed, when convection is the dominating thermal channel, a variation of the fluid velocity and flow-rate will have the largest effect on the heater temperature. Consequently, conductive heat leakage through the substrate should be reduced in order to increase the convective transfer weight, hence the sensor sensitivity and reduce the power consumption. In general, low thermal conductivity substrates are used for this purpose [14]. In some cases, the substrate material cannot be optimized with regard to thermal considerations due to external constraints such as the compatibility of the device with the operating medium or with system integration constraints.

In this work, we report on a comparative study of three micro-machined thermal flow-rate sensors made on different substrates and operated in the anemometric mode. We focus on the triple constraint of a large thermal conductivity substrate, silicon for instance, low power consumption and a large sensitivity. We report in particular an experimental and numerical comparison of the three devices. 
The paper is organized as follows: first, the devices design and fabrication process are presented. Then, the experimental setup and numerical methods are described. Finally, the major results regarding the devices performances, sensitivity and power consumption in particular, are reported and discussed. The advantages and weaknesses of each configuration are highlighted and further improvements are suggested.

\section{METHODS}

\subsection{Sensors Design and Fabrication}

The three sensors have in common the sensing element made of three 4-probe platinum resistors. Platinum wires are 106- $\mu \mathrm{m}$ long, $10-\mu \mathrm{m}$ wide and 340-nm thick. Platinum is chosen as a material for its high TCR, which is $2.218 \times 10^{-}$ ${ }^{3}{ }^{\circ} \mathrm{C}^{-1}$ in our case, and the excellent linearity of its resistivity temperature dependence. There are some other materials that have higher TCR value than platinum $(\mathrm{Pt})$, but none of them show high sensitivity, reproducibility and chemical stability at high temperature [15]. Besides, this material has corrosion resistance and outstanding accuracy with approximately one order of magnitude uncertainty [11], [16]-[18]. The use of Platinum as a resistor material is predominant in this research field [19], along with the employment of several other metals and mixtures such as platinum/palladium [20], platinum/titanium [21], platinum/chromium [22], platinum/aluminum [23] and chromium/gold [24]. These metals are used to fabricate the heater resistor for a variety of flow-rate sensors for different operation media.

The sensors under consideration are fabricated on three different substrates: glass, silicon and a silicon membrane. These three configurations will be referred to as Gl-sensor, Si-sensor and Mb-sensor, respectively. Three resistors are deposited on each substrate to enable the different operating modes previously mentioned in the introduction: calorimetric, anemometric and TOF (Time-of-Flight). Only one resistor is used in the present study. we operate according to the anemometric mode.

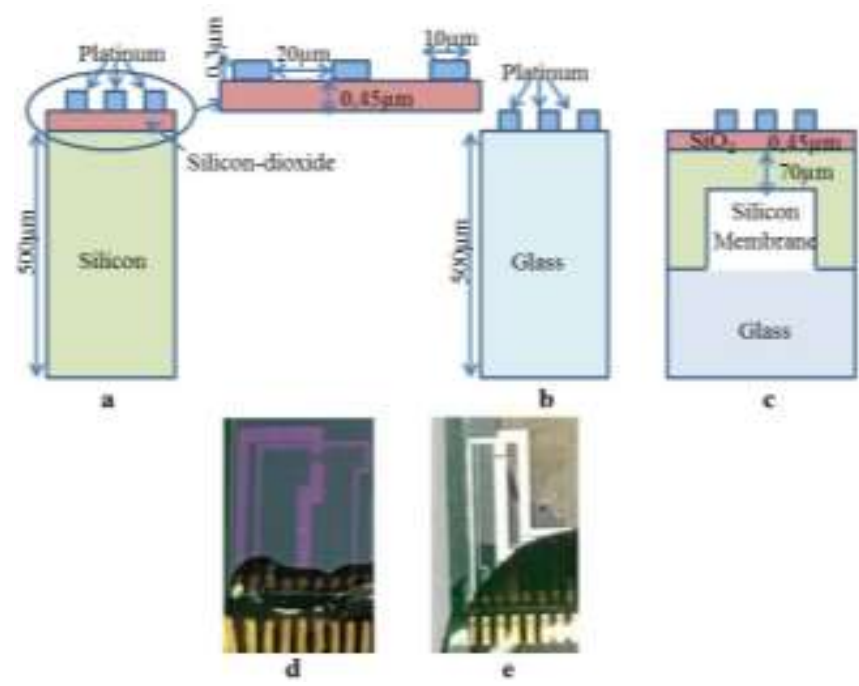

Fig. 1 Schematic of (a) silicon, (b) glass, and (c) membrane structure flow rate sensor; fabricated flow-rate sensor on (d) silicon substrate and (e) glass substrate.

The geometric properties of the three configurations are presented in Fig. 1. When the substrate is not made of glass (Fig. 1-a,c), a $0.45 \mu \mathrm{m}$-thick $\mathrm{SiO}_{2}$ insulation layer is introduced between the resistors and the $500 \mu \mathrm{m}$-thick silicon substrate or the silicon membrane to reduce the overall device thermal conductance. $\mathrm{The} \mathrm{SiO}_{2}$ layer is produced by thermal wet oxidation. The Pt resistors are patterned by a lift-off process following sputter-deposition. For Mb-sensor (Fig. 1-c), the Pt resistors are mounted on a silicon membrane resulting from backside silicon etching. The cavity depth of the membrane is nearly $430 \mu \mathrm{m}$. Glass is used as a support under the silicon membrane.

In order to perform the experiments, the sensor chip is connected to an electric circuit for power supply and the sensor's response reading. A T-shaped PCB is designed for this purpose. On the top of the PCB, a $1 \times 1 \mathrm{~cm}$ space is created to place the chip and the electrical wire bonds are built using gold between the sensor's connection pads and the PCB. The height of the PCB including the base is $7.2 \mathrm{~cm}$ and the width of the body part excluding the base is $1.18 \mathrm{~cm}$. The height and width of the base itself is $0.89 \mathrm{~cm}$ and $3.82 \mathrm{~cm}$, respectively, as shown in Fig. 2. 


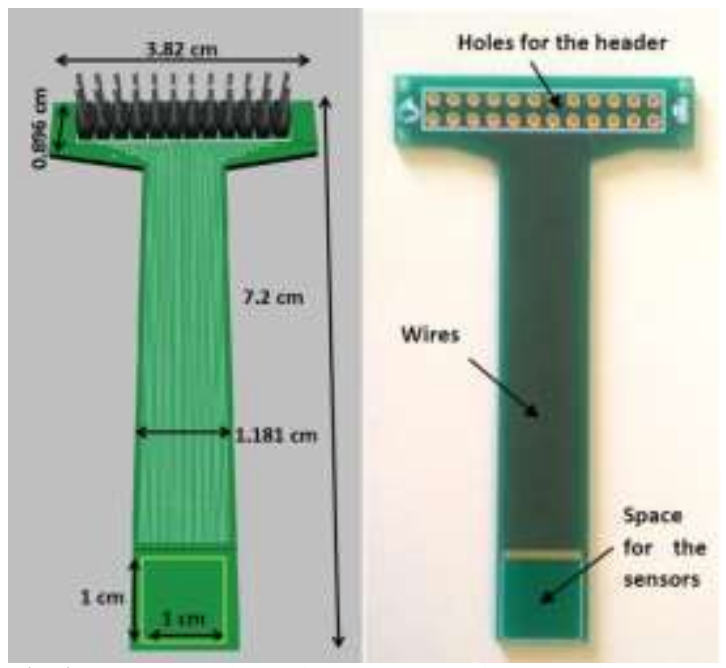

Fig. 2 The PCB designed for the sensor chip connection.

An adhesive for glass and plastic materials is used to attach the sensor chip and the PCB. Finally, the wire bonds are hermitically protected using UV-cured epoxy against water to avoid electrical short-circuit.

\subsection{Experimental Setup}

The sensor is inserted into a $25-\mathrm{mm}$ diameter PVC pipe. The pipe length is 10 times larger than its diameter to ensure an established flow regime at the sensor's position and avoid edge effects. The fluid flow is created by a variable speed pump, which is submerged in a 20-liter water reservoir and operated in closed loop. A schematic and picture of the experimental setup are shown in Fig. $3 \mathrm{a}$ and b, respectively.
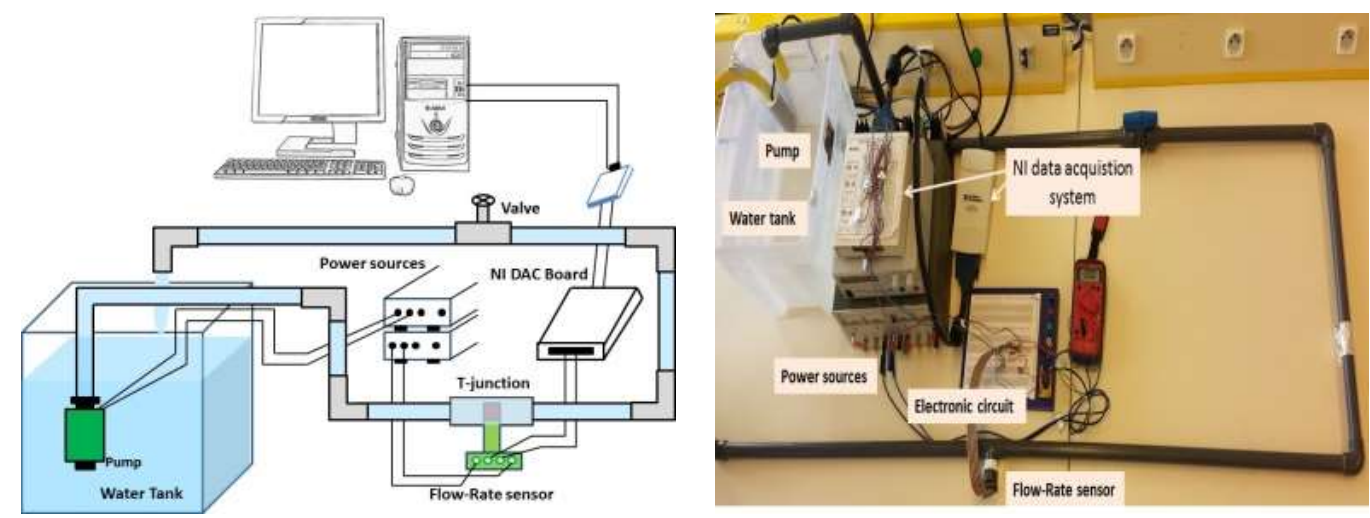

b

Fig. 3 Schematic (a) and picture (b) of the used experimental setup.

Hot-wire anemometric operation scheme is employed to extract the sensor's response to fluid velocity. To ensure a proper operation under the anemometric mode, a constant current source is used to supply the current to the 4-probe heater-resistor. LabView software interface is used for data acquisition. For each flow-rate value, the resistance drop due to the temperature variation caused by flow cooling is measured. Comparison of the resistance value with zerovelocity situation provides access to the fluid velocity using tabulated data obtained through a calibration process.

\subsection{Simulation}

A computational fluid dynamics numerical analysis based on finite elements method is carried out to extract the effect of different parameters, of both geometry and used materials, on the sensor performances. COMSOL Multiphysics is used to perform the numerical study using conjugate heat transfer module; to account for heat transfer both in solid and fluid media as well as the fluid transport. The geometry as presented in Fig. 1a, b and c is used to 
build the numerical simulation domain shown in Fig. 4. All used materials physical properties are imported from COMSOL Material Library. A parametric study is conducted for each configuration in order to study the temperature variation under a water flow at different velocities for a given supplied power. The considered sensors are sensitive to the velocity. Therefore, they are placed in the middle of the simulation domain to experience the maximum velocity. The fluidic domain is 6 times larger in width and 3 times larger in height than the chip size to minimize edge effects and the effect of boundary conditions on the sensor.

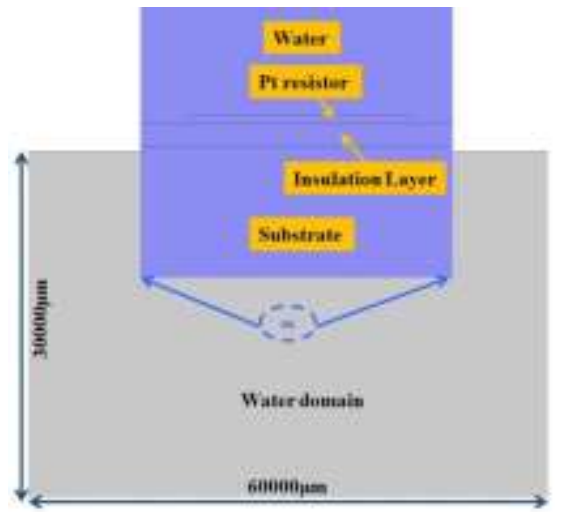

Fig. 4 Schematic representation of the numerical simulation domain.

\section{RESULTS AND DISCUSSION}

An anemometric flow rate sensor measures a temperature drop due to a fluid flow. The velocity is then deduced from the cooling magnitude. Consequently, the sensor Joule self-heating under non-flowing fluid is a critical parameter. Joule self-heating experiment enables the prediction of the sensor behavior and sensitivity without performing further experiments.

\subsection{Joule self-heating and Device turndown}

We plot in Fig. 5 the temperature $\Delta \mathrm{T}_{\max }$ of a platinum resistor under no flow for different supply currents and for the different prototypes, where the $\Delta \mathrm{T}_{\max }$ is the difference between the heater and the ambient temperature under noflow condition. For a $30 \mathrm{~mA}$ supply current, Gl-sensor exhibits a temperature increase by $11.66^{\circ} \mathrm{C}$ larger than the two other devices. As a result, we expect Gl-sensor to be more sensitive to a wider flowrate range and less power consuming under the same operating conditions than the two other prototypes.

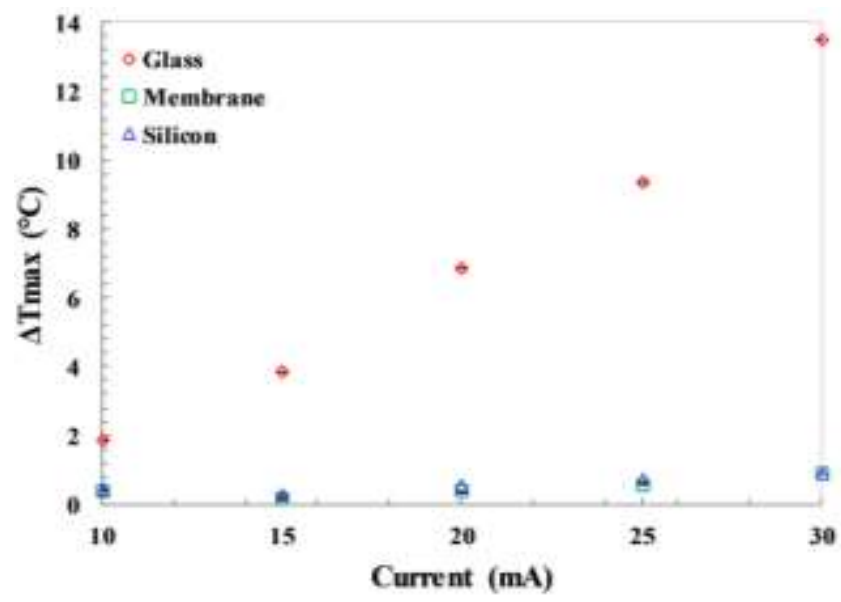

Fig. 5 Heater-resistor's temperature under no-flow condition at different supply current intensities. Error bars are plotted but cannot be distinguished because of small uncertainties. 
The sensitivity difference is expected to be more critical when low power consumption is targeted. Furthermore, Joule self-heating results provide information regarding the minimal operating current of a given device. For instance, according to Fig. 5 results, Gl-sensor can be properly operated under supply currents as low as $15 \mathrm{~mA}$. On the other hand, operating the Si-sensor and $\mathrm{Mb}$-sensor at a supply current lower than $25 \mathrm{~mA}$ would be difficult. The temperature increase due to Joule self-heating, $\Delta \mathrm{T}_{\max }$, is close to zero for these two sensors at supply current smaller than $25 \mathrm{~mA}$. As a consequence, the $\mathrm{Si}$ and $\mathrm{Mb}$-sensor deliver inconsistent responses for velocities smaller than $0.6 \mathrm{~m} / \mathrm{s}$ as shown in Fig. 6 where the sensors' relative temperature (RT) drop is plotted versus the flow velocity. The RT drop is defined as,

$$
R T=\frac{\Delta T}{\Delta T_{\max }}
$$

Where, $\Delta T=T_{h, v}-T_{a}$ and $\Delta T_{\text {max }}=T_{h, 0}-T_{a}$. Here, $T_{h, v}$ and $T_{h, 0}$ is the heater temperature at velocity $v$ and at zero velocity respectively; and $T_{a}$ is the ambient temperature. The value of $\Delta \mathrm{T} / \Delta \mathrm{T}_{\max }$ quantifies the fluid flow cooling effect which provides information on the sensor sensitivity level and turndown ratio as well. The smaller the value of $\Delta \mathrm{T} / \Delta \mathrm{T}_{\max }$, the more sensitive the sensor. However, when $\Delta \mathrm{T} / \Delta \mathrm{T}_{\max }$ reaches zero for a given velocity, the sensor is saturated for larger velocities. In other words, $\Delta \mathrm{T} / \Delta \mathrm{T}_{\max }$ ranges between 1 and 0 . For the former, the fluid velocity is not large enough to be sensed. For the latter, the sensor is saturated and any further increase of the velocity is not sensed.

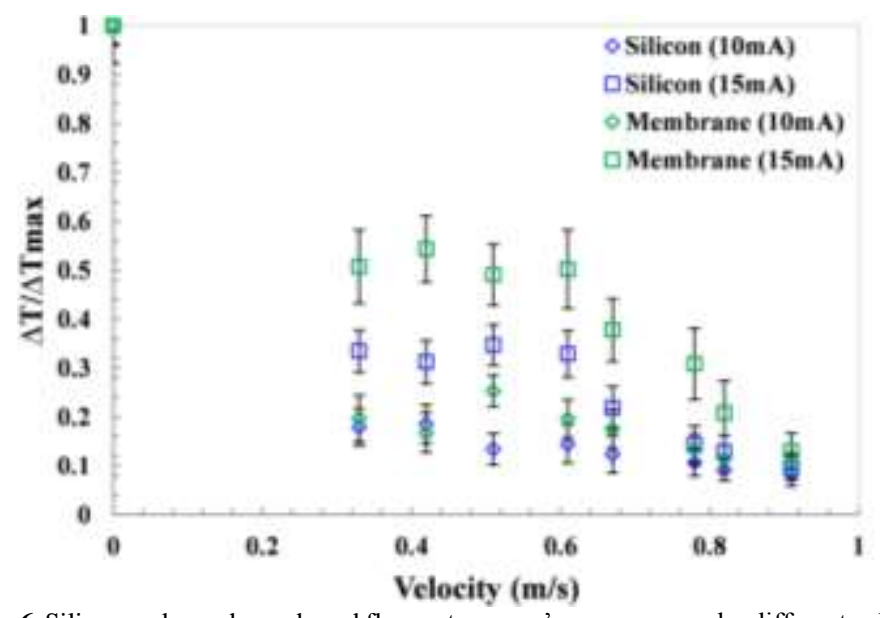

Fig. 6 Silicon and membrane based flow-rate sensor's responses under different velocities at 10 and $15 \mathrm{~mA}$ supply current.

According to Fig. 6, we observe no significant variation of the sensors temperature when the velocity increases up to $0.6 \mathrm{~m} / \mathrm{s}$. At $10 \mathrm{~mA}$, the problem persists for larger velocities. For a $15 \mathrm{~mA}$ supply current, a significant cooling of the devices is observed for velocities larger than $0.6 \mathrm{~m} / \mathrm{s}$. 


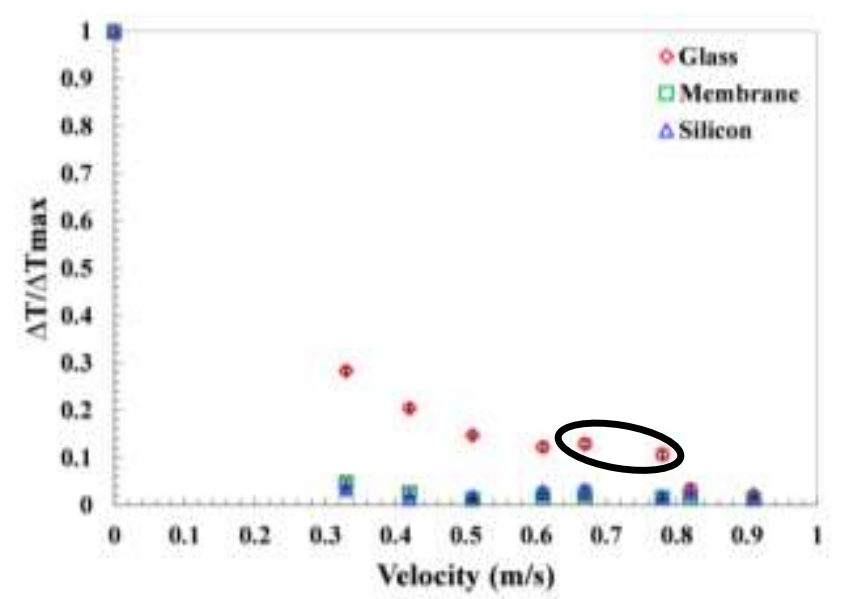

Fig. 7 Glass, membrane and silicon flow-rate sensors responses at $30 \mathrm{~mA}$ under different velocities.

On the other hand, at $15 \mathrm{~mA}$ supply current, the membrane and silicon substrate based sensors are saturated at 0.42 $\mathrm{m} / \mathrm{s}$ since no temperature variation is observed for larger velocities as shown in Fig. 7. This in not observed for Glsensor. Therefore, the sensors' behaviors expected from simple Joule heating experiments (Fig. 5) are in agreement with experiments under a variable velocity flow (Fig. 7).

\subsection{Sensitivity}

We performed numerical simulations for the three considered flow-rate sensors to enable a quantitative assessment of the devices' sensitivity, both numerically and experimentally. Results are plotted in Fig. 8. Numerical simulations are done over a wide velocity range at different power supply values. Experimental data are recorded for different supply currents under a velocity range from $0.33 \mathrm{~m} / \mathrm{s}$ to $0.91 \mathrm{~m} / \mathrm{s}$ because of experimental constraints on the reachable velocity values.

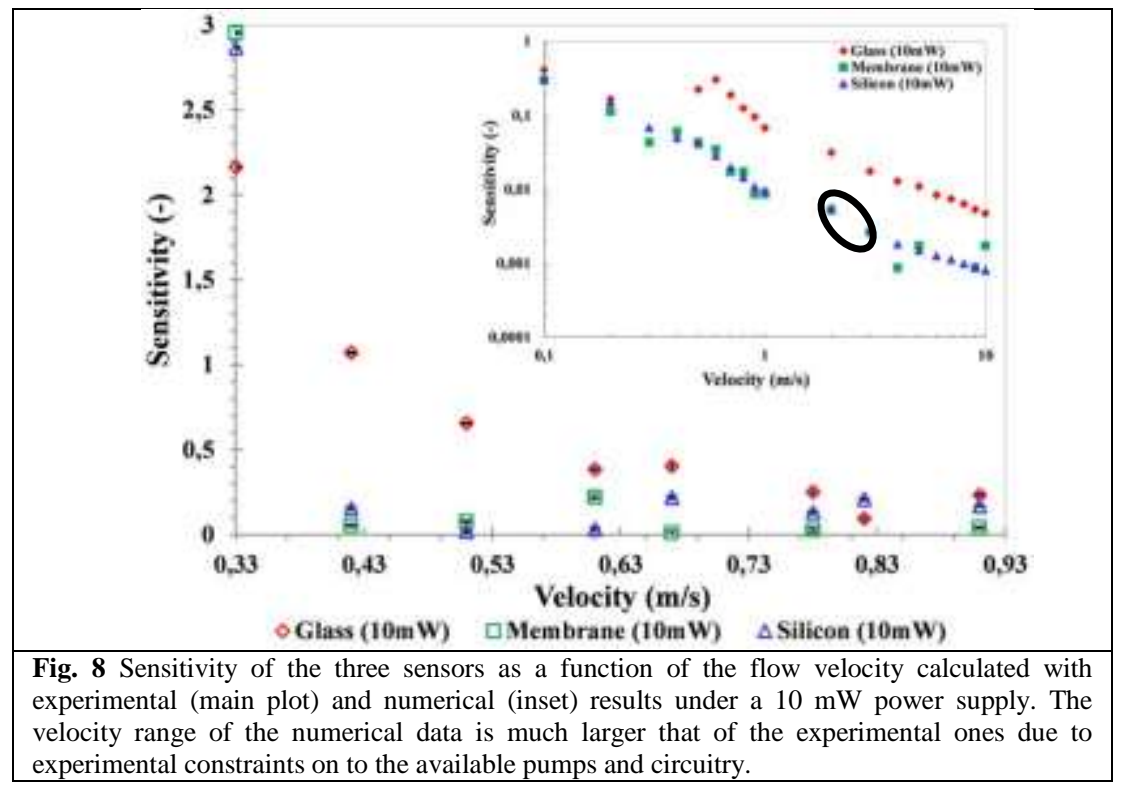

For both cases, the sensors sensitivity was calculated at $10 \mathrm{~mW}$ power supply. The sensitivity is defined as the ratio between the non-dimensional temperature variations with respect to the velocity changes: 


$$
\text { Sensitivity }=\frac{\left(\frac{\Delta T}{\Delta \operatorname{TTax}}\right)_{n}-\left(\frac{\Delta T}{\Delta \operatorname{Tmax}}\right)_{n+1}}{(\text { velocity })_{n+1}-(\text { velocity })_{n}}
$$

Where $n$ is the sequence number of the velocities and the corresponding relative temperature variations.

Sensitivity trends as a function of velocity obtained by experimental (Fig. 8) and numerical (Fig. 8 inset) data are consistent. The Gl-sensor shows a larger sensitivity for both low and high velocity values than the Si-sensor and Mbsensor. The main substrate material property that governs the sensor's response is thermal conductivity. Glass has low thermal conductivity compared to silicon, hence a better thermal insulation. As a result, numerical and experimental results show Gl-sensor is better than the silicon and membrane based sensors with respect to sensitivity and power consumption. If another insulator is used instead of glass then the sensor's response will depend on its thermal conductivity. For a larger thermal conductivity, the sensor's performance will decrease. On the opposite, for lower thermal conductivities, the sensor performance will be improved. A numerical study is conducted to assess this dependency, where glass is used as substrate material but its thermal conductivity is varied. The standard value of quartz glass thermal conductivity in COMSOL Materials Library is $1.4 \mathrm{~W} / \mathrm{m}$.K. Simulations are performed with two different thermal conductivity values, $0.014 \mathrm{~W} / \mathrm{m} . \mathrm{K}$ and $14 \mathrm{~W} / \mathrm{m} . \mathrm{K}$; which can be considered as insulating materials 1 and 2 respectively. Numerical results are presented in Fig. 9. The glass substrate device with standard thermal conductivity $(1.4 \mathrm{~W} / \mathrm{m} . \mathrm{K})$ shows an intermediate response in comparison with insulator 1 and 2 . The device with low thermal conductivity substrate $(0.014 \mathrm{~W} / \mathrm{m} . \mathrm{K})$ exhibits a high sensitivity in the whole considered velocity range. Besides, the RT of insulator 2 flow-rate sensor with as substrate goes to zero and reaches saturation very rapidly; which indicates a faster cooling of the heater and a small device's turn-down ratio.

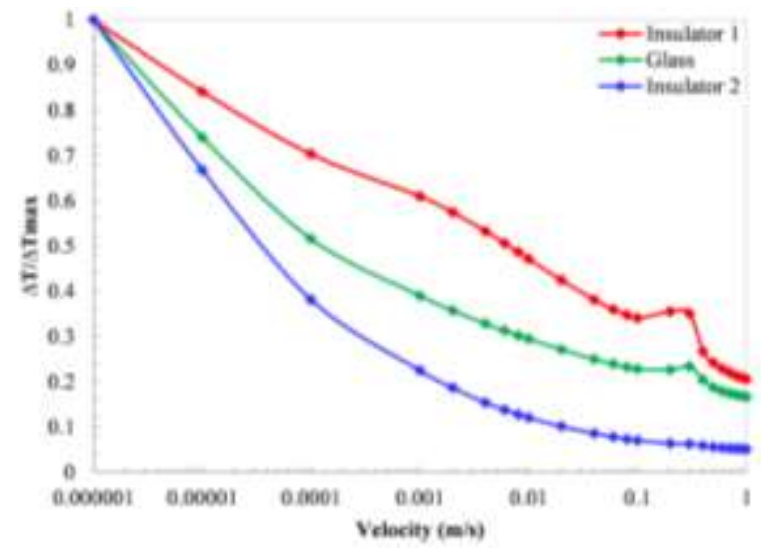

Fig. 9 Normalized temperature variation of the flow-rate sensor at different velocities when different hypothetical insulating materials are used instead of glass as substrate.

We also observe that, at a given velocity range, around $0.6 \mathrm{~m} / \mathrm{s}$, the sensitivity of the Gl-sensor is not following the monotonous decreasing trend (Fig. 8 inset). The same non-monotonic behavior can be observed in Fig. 7 and Fig. 9 for Gl-sensor on the normalized temperature decrease plot. This unexpected behavior is discussed in the following paragraph.

\subsection{The response non-monotonicity and outliers}

In Fig. 7, Fig. 8 inset and Fig. 9 a non-monotonic variation is observed of the Gl-sensor temperature when the fluid velocity increases around $0.6 \mathrm{~m} / \mathrm{s}$. Such a behavior is not expected since an increase of the flow velocity, induces a larger convective cooling, hence a smaller heater temperature.

The velocity field around the device is plotted in Fig. 10. The plotted field shows a reverse flow (opposite to the main flow velocity direction) at the vicinity of the resistor. This reverse flow carries back downstream hot fluid which, instead of cooling the resistor, heats it up. The same velocity field, with a reverse flow in particular, can be observed for Si-sensor and $\mathrm{Mb}$-sensor. However, due to their lower Joule self-heating, downstream fluid brought back by the reverse flow is not hot enough in this case to induce an increase of the sensor temperature. In addition, the reverse flow is observed for velocities larger than $0.6 \mathrm{~m} / \mathrm{s}$ while Si-sensor and $\mathrm{Mb}$-sensor are saturated starting from velocities as small as $0.4 \mathrm{~m} / \mathrm{s}$. 


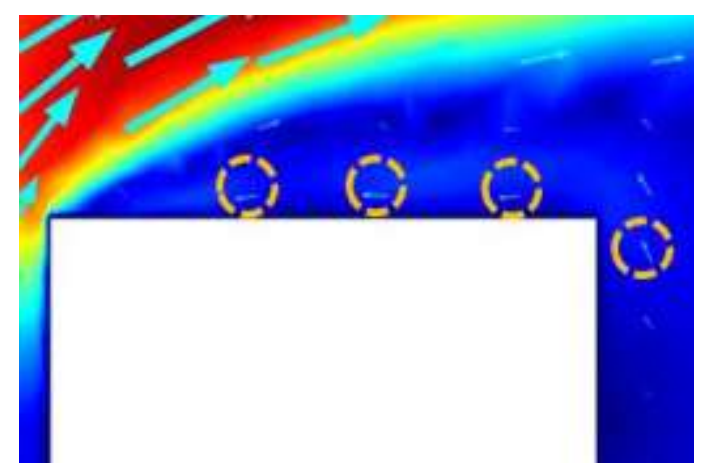

Fig. 10 Reverse flow at the sensor's heater-resistor vicinity at $0.3 \mathrm{~m} / \mathrm{s}$ and $0.4 \mathrm{~m} / \mathrm{s}$.

The reverse flow and the resulting non-monotonic evolution of Gl-sensor can be detrimental for an accurate measurement of the fluid velocity and flowrate. Indeed, the fluid velocity is deduced from the sensed temperature. A "wrong" temperature measurement leads to an error in the calculated velocity value. We can observe in Fig. 7 that the same temperature is measured for two different fluid velocities around $0.6 \mathrm{~m} / \mathrm{s}$. With no additional information, the two velocity values can-not be discriminated. Such problems can be avoided by a proper optimization of the sensor chip shape, the edges curvature for instance, which significantly reduces the reverse flow magnitude, hence the resulting flow-rate measurements discrepancies. This solution has been validated by numerical simulations. Results are not shown here.

\subsection{Power consumption}

According to the previous paragraphs and the sensitivity assessment of the three sensors, Gl-sensor exhibits higher performances. In addition to large sensitivity, low power consumption is a critical criterion for the studied devices which are developed as components of low power sensor networks. A comparative study of the three sensors power consumption is conducted. Power consumption is calculated using Ohm's law: $\mathrm{P}=\mathrm{IR}^{2}$. Supply current intensity is varied from $10 \mathrm{~mA}$ to $30 \mathrm{~mA}$ with a step of $5 \mathrm{~mA}$ and the resultant Joule self-heating is assessed through the heating resistance value measurement for the three devices. The test is conducted under constant current, so the power consumption is proportional to the resistance value which varies due to the resistor's temperature variation. We plot in Fig. 11, power consumption of the three devices as a function of the supply current under zero velocity flow.

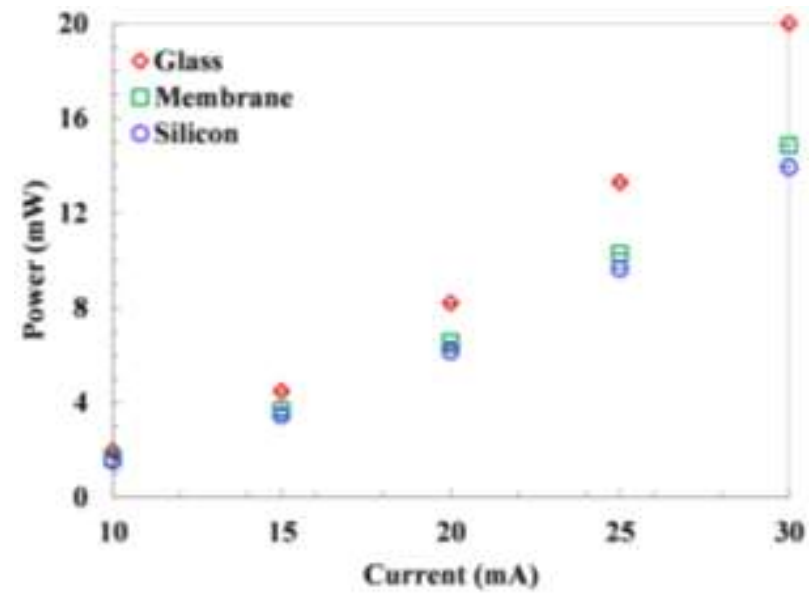

Fig. 11 Power consumption of the three sensors at zero velocity for different supply currents

The presented results show that the glass sensor is nearly 1.4 times more power consuming at maximum supply current, $30 \mathrm{~mA}$ for instance, than the membrane and silicon sensors. Such a result is surprising at first glance. Indeed, the three devices heaters are made of the same platinum resistances with identical geometries. Consequently, their power consumption under a constant current is expected to be the same. Such an expectation does not account for the platinum resistance value temperature dependence. Indeed, the larger consumption of Gl-sensor is mainly due to its better thermal insulation, hence to its larger Joule self-heating. For a given supply current, the glass substrate low thermal conductivity 
ensures larger insulation of the heater, hence a larger resistance temperature. Since the resistance value is temperature dependent, a larger temperature induces a larger resistance value, hence a larger power consumption. The drawback of the Gl-sensor larger power consumption at a given electric current can be balanced by the possibility of a proper operation of this sensor at lower supply currents, as low as $20 \mathrm{~mA}$, which in turn reduces the power consumption. Results presented in Fig. 8 are obtained for a supply current slightly larger than $20 \mathrm{~mA}$.

The numerical and experimental study presented in this paper shows that the substrate thermal conductance is the main parameter governing $\Delta \mathrm{T}_{\max }$ and $\Delta \mathrm{T} / \Delta \mathrm{T}_{\max }$ for a given power supply value, hence the sensor sensitivity. Since, silicon thermal conductivity is much larger than that of glass, around 100 times larger for instance, Gl-sensor Joule self-heating and sensitivity overcome those of silicon based sensors. Although the introduced silicon membrane diminishes the conductive heat loss through the substrate, the sensor performances enhancement is not significant. Consequently, a possible solution to obtain a sensitive and low power anemometric flow-rate sensor on a silicon substrate is to further increase the thermal insulation of the heating/sensing resistor through a geometric optimization.

\section{CONCLUSION}

Three micro-machined flow-rate sensors for autonomous water network monitoring devices have been designed, fabricated and experimentally characterized. Their main difference appears in the used substrate material: glass, bulk silicon and a silicon membrane. We show experimentally and numerically that the glass substrate device is far more sensitive and less power consuming. We also show that the key parameter for such performances is its low thermal conductivity. However, large thermal conductivity materials such as silicon can be needed for co-integration of different sensors in lab-on-chip devices which increases power consumption and reduces sensitivity. We show that a simple geometric optimization such as the use of a silicon membrane is insufficient to overcome this problem. More complex geometries and strategies for the overall device thermal conductance reduction are then needed to obtain more energy efficient and sensitive devices.

\section{ACKNOWLEDGEMENT}

This work has received funding from the European Union's H2020 Programme for research, technological development and demonstration under grant agreement No644852. Part of this work had the support of the National Research Agency (ANR) in the frame of the EquipEx project SENSE-CITY of the Programme Investissement d'Avenir (PIA), involving IFSTTAR and ESIEE Paris as founding members of the consortium. Fabrication of the chips was done in the cleanroom facilities of ESIEE Paris, whose technical staff is deeply acknowledged. Authors would like to thank H. Regina and S. Sarkar for valuable help on numerical simulations.

\section{References}

[1] S. Silvestri and E. Schena, "Micromachined Flow Sensors in Biomedical Applications," Micromachines, vol. 3, no. 2, pp. 225-243, Mar. 2012.

[2] N.-F. Chiu, T.-C. Hsiao, and C.-W. Lin, "Low power consumption design of micro-machined thermal sensor for portable spirometer," Tamkang J. Sci. Eng., vol. 8, no. 3, pp. 225-230, 2005.

[3] J. T. W. Kuo, L.-Y. Chang, P.-Y. Li, T. Hoang, and E. Meng, "A microfluidic platform with integrated flow sensing for focal chemical stimulation of cells and tissue," Sens. Actuators B Chem., vol. 152, no. 2, pp. 267276, Mar. 2011.

[4] G. Soundararajan, M. Rouhanizadeh, H. Yu, L. DeMaio, E. S. Kim, and T. K. Hsiai, "MEMS shear stress sensors for microcirculation," Sens. Actuators Phys., vol. 118, no. 1, pp. 25-32, 2005.

[5] L.-Y. Chang, P.-Y. Li, L. Zhao, T. Hoang, and E. Meng, "Integrated flow sensing for focal biochemical stimulation," in 2008 3rd IEEE International Conference on Nano/Micro Engineered and Molecular Systems, 2008, pp. 921-926.

[6] T. K. Hsiai et al., "Micro Sensors: Linking Real-Time Oscillatory Shear Stress with Vascular Inflammatory Responses," Ann. Biomed. Eng., vol. 32, no. 2, pp. 189-201, Feb. 2004.

[7] L. Billmann and R. Isermann, "Leak detection methods for pipelines," Automatica, vol. 23, no. 3, pp. 381-385, May 1987.

[8] K. H. Bartlett, M. Martinez, and J. Bert, "Modeling of Occupant-Generated CO2 Dynamics in Naturally Ventilated Classrooms," J. Occup. Environ. Hyg., vol. 1, no. 3, pp. 139-148, Mar. 2004.

[9] Y. Zhu, B. Chen, M. Qin, and Q. A. Huang, "2-D Micromachined Thermal Wind Sensors \#x2014;A Review," IEEE Internet Things J., vol. 1, no. 3, pp. 216-232, Jun. 2014. 
[10] G.-P. Shen, M. Qin, Q.-A. Huang, H. Zhang, and J. Wu, "A FCOB packaged thermal wind sensor with compensation," Microsyst. Technol., vol. 16, no. 4, pp. 511-518, Apr. 2010.

[11] N. T. Nguyen, "Micromachined flow sensors-a review," Flow Meas. Instrum., vol. 8, no. 1, pp. 7-16, Mar. 1997.

[12] J. T. W. Kuo, L. Yu, and E. Meng, "Micromachined Thermal Flow Sensors-A Review," Micromachines, vol. 3, no. 3, pp. 550-573, Jul. 2012.

[13] R. Vilares et al., "Fabrication and testing of a SU-8 thermal flow sensor," Sens. Actuators B Chem., vol. 147, no. 2, pp. 411-417, Jun. 2010.

[14] W.-C. Lin and M. A. Burns, "Low-power micro-fabricated liquid flow-rate sensor," Anal. Methods, vol. 7, no. 9, pp. 3981-3987, Apr. 2015.

[15] F. Mailly et al., "Anemometer with hot platinum thin film," Sens. Actuators Phys., vol. 94, no. 1-2, pp. 32-38, Oct. 2001.

[16] J. van Honschoten, J. van Baar, H. E. de Bree, T. Lammerink, G. Krijnen, and M. Elwenspoek, “Application of a microflown as a low-cost level sensor," J. Micromechanics Microengineering, vol. 10, no. 2, p. $250,2000$.

[17] D. Dittmann, R. Ahrens, Z. Rummler, K. Schlote-Holubek, and W. K. Schomburg, "Low-Cost Flow Transducer Fabricated with the Amanda-Process," in Transducers '01 Eurosensors XV, Springer, Berlin, Heidelberg, 2001, pp. 1444-1447.

[18] K. G. Kreider and F. DiMeo, "Platinum/palladium thin-film thermocouples for temperature measurements on silicon wafers," Sens. Actuators Phys., vol. 69, no. 1, pp. 46-52, Jun. 1998.

[19] R.-H. Ma, D.-A. Wang, T.-H. Hsueh, and C.-Y. Lee, “A MEMS-Based Flow Rate and Flow Direction Sensing Platform with Integrated Temperature Compensation Scheme," Sensors, vol. 9, no. 7, pp. 5460-5476, Jul. 2009.

[20] M. Zanini et al., "Fabrication and properties of a Si-based high-sensitivity microcalorimetric gas sensor," Sens. Actuators Phys., vol. 48, no. 3, pp. 187-192, May 1995.

[21] R. Viard et al., "A robust thermal microstructure for mass flow rate measurement in steady and unsteady flows," J. Micromechanics Microengineering, vol. 23, no. 6, p. 065016, 2013.

[22] A. Götz et al., "A micromachined solid state integrated gas sensor for the detection of aromatic hydrocarbons," Sens. Actuators B Chem., vol. 44, no. 1, pp. 483-487, Oct. 1997.

[23] P. Norlin, O. Öhman, B. Ekström, and L. Forssén, "A chemical micro analysis system for the measurement of pressure, flow rate, temperature, conductivity, UV-absorption and fluorescence," Sens. Actuators B Chem., vol. 49, no. 1, pp. 34-39, Jun. 1998.

[24] T. S. J. Lammerink, N. R. Tas, M. Elwenspoek, and J. H. J. Fluitman, "Micro-liquid flow sensor," Sens. Actuators Phys., vol. 37, pp. 45-50, Jun. 1993. 